\title{
54. The Presence of a Sleep-promoting Material in the Brain of Sleep-deprived Rats
}

\author{
By Hiroaki NAGASAKI,*) Masami IRIKI,*) Shojiro INouÉ,**) \\ and Koji UCHIZONo***)
}

(Comm. by Yasuji Katsuki, M. J. A., March 12, 1974)

Introduction. Mechanisms of sleep have been investigated in neurological and neurochemical aspects for the past decades. In neurochemical aspect, Legendre and Piéron $(1910,1913)$ reported that transfusion of the cerebrospinal fluid (c.s.f.) from sleep-deprived dog induced behavioral sleep in recipient dog. Schnedorf and lvy's experiment (1939) confirmed this phenomenon. Monnier and Hoesli $(1964,1965)$ reported that sleep might be mediated by an extracorporal hemodialysate of donor rabbit kept asleep by electrical stimulation of the thalamic sleep center. Recently they showed further that the extracorporal hemodialysate contained a heptapeptide which gave rise to hypnogenic effect (1972). In 1971 Fencle et al. showed that sleep-promoting substance was contained in the c.s.f. of sleep-deprived goats and the sleep-promoting fraction was found in a low molecular weight fraction (mol. wt. <500).

Accordingly the sleep-promoting materials have been proved to be present in the blood and/or the c.s.f., but so far there has been no report of their presence in the brain itself. In this experiment the presence of a sleep-promoting material in the brain of sleep-deprived rats was investigated.

Methods. Sleep deprivation of rats and isolation of sleep-promoting material. In order to deprive the sleep of rats for 24 hours, an electric shock of $3.5 \mathrm{~mA}, 50 \mathrm{~Hz}$ was given to 20-30 male rats (200$300 \mathrm{~g}$ ) contained in a cage whenever any one of animals fell asleep (for details see Ichikawa et al., 1973). After 24-hour sleep deprivation, rats were sacrificed and the brain stem including the medulla oblongata, pons, mesencephalon and hypothalamus was separated. The tissues were homogenized with 5 volumes of distilled water by teflon homogenizer by several strokes. The homogenate was dialyzed

*) Department of Physiology, Tokyo Metropolitan Institute of Gerontology, 35-2 Sakaecho, Itabashi-ku, Tokyo.

**) Institute for Medical and Dental Engineering, Tokyo Medical and Dental University, 2-3-10 Surugadai, Kanda, Chiyoda-ku, Tokyo.

***) Department of Physiology, Faculty of Medicine, University of Tokyo, 7-3-1 Hongo, Bunkyo-ku, Tokyo. 
against distilled water for 5 hours in the cold room. The dialysate was lyophylized by vacuum lyophylizer, Burtis. The lyophylized materials were kept at $-74^{\circ} \mathrm{C}$ before use. About 1000 rats were used for the isolation of sleep-promoting material.

Examination of sleep-promoting effect. The sleep-promoting effect was examined by the reduction of locomotor activities and an increase of a quantity of delta waves (1-4 Hz) of EEG of the recipient male rat. The locomotor activities were measured by ANIMEX, Farad, Activity meter type-SE. EEG was recorded on a polygraph, Nihon-kohden, model-RM5, using the telemetry system (Transmitter, Narco, model-FM100E3 and Biotelemetry Receiver, Narco, modelFM1006). The quantity of delta waves in EEG in each 10-second interval was analyzed by an automatic frequency analyzer with bandpass filter and recorded on custom demand recorder, Toa, modelCDR12A (for details see Asaki et al., 1974). The total sleep time in 24 hours was calculated from the sum of the sleep time that is the length from the onset to the end of the appearance of delta waves. The examination of sleep-promoting effect was performed 2-4 days after the surgical operation for EEG electrode implantation because the effect of operation disappeared within 2-4 days. The room temperature was kept at $25^{\circ} \mathrm{C}$ and the daily lighting regimen was from $8: 30-9: 30$ to $18: 30-19: 30$. The brain extract was intraperitoneally injected without anesthesia at 12:00. In all experiments the dose of an injected material was 6 units dissolved in $0.3 \mathrm{ml}$ of $\mathrm{H}_{2} \mathrm{O}$. 1 unit was an equivalent amount of the active material extracted from one sleep-deprived or control rat.

Results. 1. Effect of the brain extract on the locomotor activities of the rat. Fig. 1 shows the daily pattern of the locomotor activities in a male rat before injection (baseline, a), after an intraperitoneal injection of the brain extract from sleep-deprived rats at 12:00 (experiment, b) and after an intraperitoneal injection of the brain extract from control rats at 12:00 (control, c). The results were obtained from the same rat. The locomotor activities were integrated in each 60 minutes in this figure. This experiment demonstrates the following results; (1) The injection of the brain extract from sleep-deprived rats reduces the locomotor activities of the rat. As shown in Fig. 1b this reduction is especially evident during the midnight, when the locomotor activities are otherwise the highest in this species of animals. (2) The injection of the brain extract from control rats has no such effect on the locomotor activities of the rat. As shown in Fig. 1c the pattern of daily locomotor activities of the control recording was the same as that of the baseline recording, that 


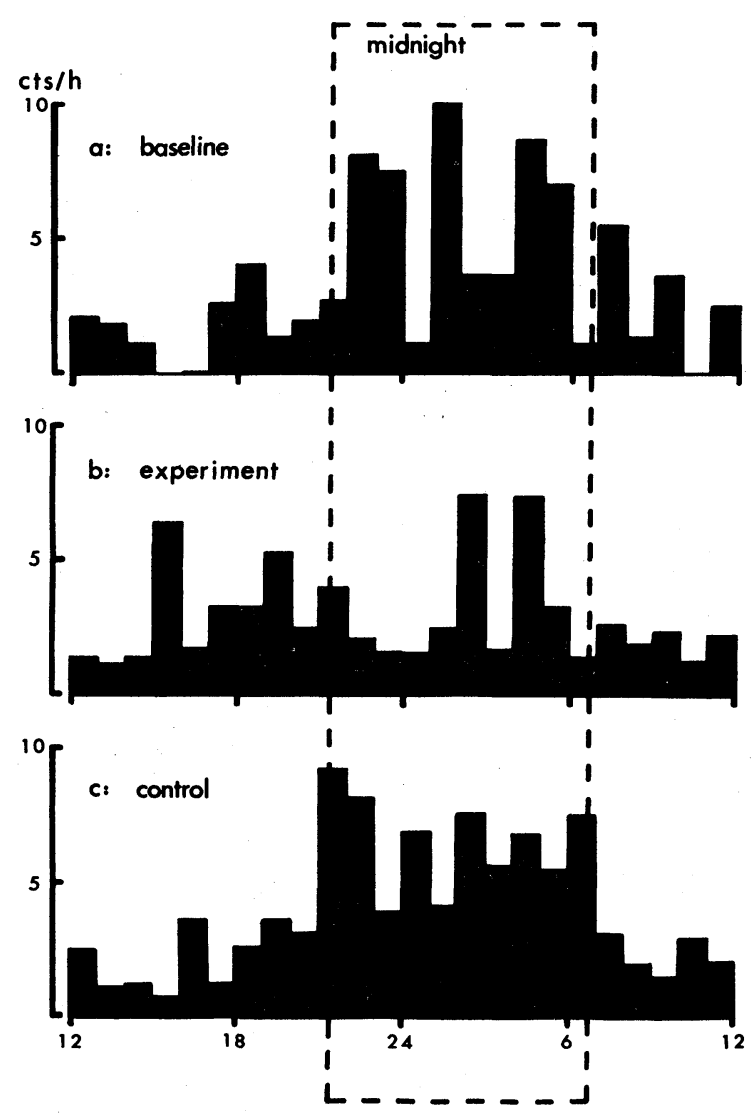

Fig. 1. Daily pattern of the locomotor activities in a male rat before (a: baseline) and after (b: experiment) an intraperitoneal injection of the brain extract from sleep-deprived rats and control rats (c: control) without anesthesia at 12:00 with 6 units/ $0.3 \mathrm{ml}$ of $\mathrm{H}_{2} \mathrm{O}$.

Ordinate: the locomotor activities, counts/hour. Abscissa: time in hour. The brain extract from sleepdeprived rats remarkably reduces the locomotor activities, especially at midnight (b), but the brain extract from control rats has no such effect (c). Compare $\mathrm{a}, \mathrm{b}$ and $\mathrm{c}$ in the dashed rectangle.

is, the locomotor activities were high during the night and low during the daytime. From the fact that the injection of the brain extract from control rats had no reducing effect on the locomotor activities of the rat, the nonspecific reducing effect which might be produced by injection of the brain extract, does not need to be considered. The reduction of the daily locomotor activity due to the intraperitoneal injection of the brain extract from sleep-deprived rats was confirmed 
in 3 experiments. In all experiments the daily locomotor activities were reduced. The reduction rates were $17.9 \%, 35.5 \%$ and $12.7 \%$ respectively, and the average and standard error in all 3 experiments was $22.0 \pm 9.6 \%$.

2. Effect of the brain extract on the quantity of delta waves

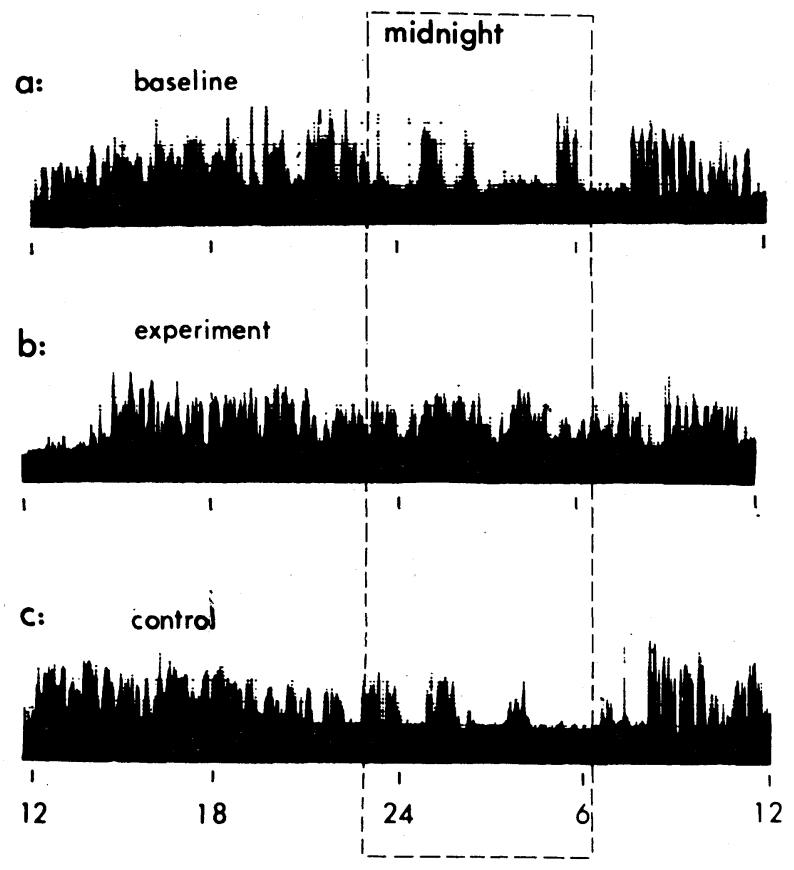

wake
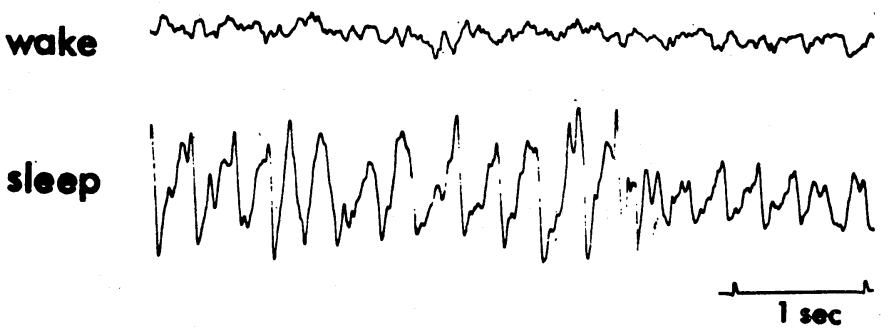

Fig. 2. Daily pattern of the quantity of delta waves in a male rat before (a: baseline) and after (b: experiment) an intraperitoneal injection of the brain extract from sleep-deprived rats and control rats (c: control) without anesthesia at 12:00 with 6 units $/ 0.3 \mathrm{ml}$ of $\mathrm{H}_{2} \mathrm{O}$.

Ordinate: the quantity of delta waves per 10 seconds. Abcissa: time in hour. An injection of the brain extract from sleepdeprived rats increased the quantity of delta waves, especially at midnight (b). On the contrary, the brain extract from control rats had no significant effect (c). Compare $a, b$ and $c$ in the dashed rectangle. Telemetricaly recorded EEGs are shown below during wake and sleep of the rat. 
in $E E G$ of the rat. Fig. 2 demonstrates the daily pattern of the quantitative change of delta waves in EEG. All recordings were performed in the same male rat. As shown in the baseline recording (Fig. 2a), i.e., before injection, delta waves appeared more frequently during the daytime when the rat tends to sleep, and less frequently during the night when the rat tends to wake. After an intraperitoneal injection of the brain extract from sleep-deprived rats at 12:00 (Fig. 2b) the quantity of delta waves increased, indicating an increase of sleep. The increase of delta waves was especially remarkable during the midnight, with the concomitant reduction of the locomotor activities as shown in Fig. 1b. On the contrary, the injection of the brain extract from control rats had no significant effect on the quantitative change of delta waves in the control recording as shown in Fig. 2c. This fact indicates that, concerning the increase in delta waves in EEG, the nonspecific effect of the brain extract does not need to be considered.

Table I. Effect of the brain extract on the total sleep time of male rats

\begin{tabular}{cccc}
\hline baselin & \multicolumn{2}{c}{ experiment } & control \\
$24 \mathrm{hr}$ & 1st $24 \mathrm{hr}$ & 2nd $24 \mathrm{hr}$ & 1st $24 \mathrm{hr}$ \\
\hline 55.6 & 72.0 & 53.2 & 52.4 \\
66.0 & 76.2 & 62.8 & 60.6 \\
58.5 & 74.4 & 56.4 & 62.0 \\
51.7 & 73.3 & 56.5 & 58.8 \\
\hline $58.0 \pm 5.6$ & $74.0 \pm \mathbf{1 . 5}$ & $57.2 \pm 3.5$ & $58.5 \pm 3.7$ \\
\hline
\end{tabular}

Percentage of the total sleep time in 24 hours was calculated from the sum of the sleep time that is the length from the onset to the end of the appearance of delta waves. The total sleep time increased by $27.6 \%$ in the 1st 24 hours after an intraperitoneal injection of the brain extract from sleep-deprived rats, but returned to the preinjection level in the 2nd 24 hours. On the contrary, the brain extract from control rats had no significant effect.

The results were ascertained in experiments of 4 rats, which are summarized in Table I. In these experiments the quantitative change in delta waves was recorded for 1 day before and for 2 days after an injection of the brain extract. The total sleep time was increased from $58.0 \pm 5.6 \%$ to $74.0 \pm 1.5 \%$ in the first day after the injection of the brain extract from sleep-deprived rats but returned to the preinjection level in the 2 nd day $(57.2 \pm 3.5 \%)$. There was no significant difference between the baseline group and the control group $(58.5 \pm 3.7 \%)$. 
Discussion. The reduction of the locomotor activities and the concomitant increase of delta waves in the EEG indicate that a sleeppromoting material is contained in the brain extract from sleepdeprived rats. The region of brain stem used for extraction of the active material has been reported to contain a sleep center and an ascending inhibitory system against an ascending reticular activating system (Moruzzi, 1972). The participation of the sleep-promoting material in the blood which is considered to contain the hypnogenic material (Schoenenberger et al., 1972) is negligible because the amount of the blood in the brain tissue was extremely small and the effect of the brain extract from sleep-deprived rats after careful perfusion with the saline was proved to be still evident. Furthermore the effect of this material is not a nonspecific reduction of the locomotor activities of rats as described by Fencle et al. (1971), because the effect on the locomotor activities and the quantity of delta waves were only obvious after the injection of the brain extract from sleepdeprived rats but not from normal rats. The biochemical analysis of the active substances in the dialysates is in progress our laboratories.

In summary, it is highly probable that extracts from the brain stem of sleep-deprived rats contain a sleep-promoting material.

Acknowledgment. The authors express our gratitude to Miss. M. Makino, Mr. N. Matsushita and Miss. T. Chisaki for their skillful technical assistance. Parts of expenses were defrayed from the Ministry of Education of Japan.

\section{References}

1) R. Legendre and H. Piéron: C. R. Soc. Biol. (Paris), 68, 1108 (1910).

2) - - Z. allg. Physiol., 14, 235 (1913).

3) J. G. Schnedorf and A. C. Ivy: Amer. J. Physiol., 125, 491 (1939).

4) M. Monnier and L. Hoesli: Science, 146, 796 (1964).

5) —-: Pflügers Arch. ges. Physiol., 282, 60 (1965).

6) G. A. Schoenenberger, L. B. Cueni, M. Monnier, and A. M. Hatt, Pflügers Arch., 338, 1 (1972).

7) V. Fencle, G. Koski, and J. R. Pappenheimer: J. Physiol. (Lond.), 216, 565 (1971).

8) H. Ichikawa, K. Honda, and S. Inoué: Reports Med. Den. Eng. (Tokyo Med. Den. Univ.,) , 7, 145 (1973).

9) Y. Asaki, H. Nagasaki, S. Katayama, and M. Iriki: In preparation.

10) G. Moruzzi : Ergebn. Physiol., 64, 1 (1972). 